\title{
Shared Peptides in Mass Spectrometry Based Protein Quantification
}

\author{
Banu Dost ${ }^{1}$, Nuno Bandeira ${ }^{1}$, Xiangqian $\mathrm{Li}^{2}$, Zhouxin Shen ${ }^{2}$, \\ Steve Briggs ${ }^{2}$, and Vineet Bafna ${ }^{1}$ \\ 1 Department of Computer Science and Engineering, \\ University of California, San Diego, CA 92093, USA \\ \{bdost, nbandeira, vbafna\}@cs.ucsd.edu \\ 2 Department of Biological Sciences, \\ University of California, San Diego, CA 92093, USA \\ $\{x l i, z 1$ shen, sbriggs $\}$ Oucsd.edu
}

\begin{abstract}
In analyzing the proteome using mass spectrometry, the mass values help identify the molecules, and the intensities help quantify them, relative to their abundance in other samples. Peptides that are shared across different protein sequences are typically discarded as being uninformative w.r.t each of the parent proteins.

In this paper, we investigate the use of shared peptides which are ubiquitous ( $\sim 50 \%$ of peptides) in mass spectrometric data-sets. In many cases, shared peptides can help compute the relative amounts of different proteins that share the same peptide. Also, proteins with no unique peptide in the sample can still be analyzed for relative abundance. Our paper is the first attempt to use shared peptides in protein quantification, and makes use of combinatorial optimization to reduce the error in relative abundance measurements. We describe the topological and numerical properties required for robust estimates, and use them to improve our estimates for ill-conditioned systems. Extensive simulations validate our approach even in the presence of experimental error. We apply our method to a model of Arabidopsis root knot nematode infection, and elucidate the differential role of many protein family members in mediating host response to the pathogen.
\end{abstract}

Key words: shared peptides, protein quantification, linear programming, optimization, ITRAQ, mass spectrometry.

\section{Introduction}

The analysis of the proteome using mass spectrometry involves the separation of molecules (often, enzymatically digested peptides from expressed proteins) followed by accurate measurement of mass of each molecule, termed as the massspectrum. Together with mass, the spectrum also measures peak-intensity for each molecule. For any constituent peptide from a protein sequence, its spectral intensity is a measurement of abundance, the amount of the expressed protein. However, the actual value is hard to interpret, as it depends upon a number of 
poorly understood factors, including instrument types, energetics of the process, and physico-chemical properties of the peptide itself. Consequently, it is often the relative-abundance of a peptide, measured as the ratio of intensities of a peptide across samples, that is investigated $[4,8]$. By the same token, intensity values of different peptides are usually not comparable.

The relative abundance of a peptide is a proxy for the relative abundance of the parent protein. This is acceptable only when the peptide sequence is unique to the protein. By contrast, when a peptide is shared across proteins (Ex: proteins that share domains), its abundance (and relative abundance) depends upon contributions from multiple proteins. For this reason, shared peptides have been traditionally disregarded in protein-level quantification analysis. However, this may significantly decrease the number of proteins for which abundance estimates can be obtained. While often unreported, a significant portion of the data (as much as 50\%) is ignored. In our own experiment with Arabidopsis proteins, $4,145(48 \%)$ of the 8,584 expressed proteins were not represented by a unique peptide and would normally be discarded.

The goal of this paper is to demonstrate that shared peptides are a resource that adds value, and we make the point with two simple examples. Consider a case with two proteins $p_{1}, p_{2}$, and 3 constituent peptides $s_{1}, s_{2}, s_{3}$, where $s_{1}, s_{2}$ are unique, and $s_{3}$ is shared. See Figure 1a, where a peptide is connected to a protein by an edge only if it is contained in it. Consider an experiment that revealed the relative abundances $\left(r_{1}, r_{2}, r_{3}\right)$ of the 3 peptides over two samples $B$ and $A$ as 16,1,4 respectively. The typical approach is to discard the shared peptide $s_{3}$, and to assert that $p_{1}$ is $16 \times$ over-expressed, while $p_{2}$ is unchanged. Formally, if $Q_{j}^{A}, Q_{j}^{B}$ represent the actual abundance of protein $j$ in samples $A, B$ respectively, then

$$
\frac{Q_{1}^{A}}{Q_{1}^{B}}=r_{1}=16 \text { and } \frac{Q_{2}^{A}}{Q_{2}^{B}}=r_{2}=1 .
$$

Our point is that the ignored peptide $s_{3}$ also provides information because

$$
r_{3}=4 \simeq \frac{Q_{2}^{A}+Q_{1}^{A}}{Q_{2}^{B}+Q_{1}^{B}}=\frac{Q_{2}^{B}+16 Q_{1}^{B}}{Q_{2}^{B}+Q_{1}^{B}}=\frac{16+\frac{Q_{2}^{B}}{Q_{1}^{B}}}{1+\frac{Q_{2}^{B}}{Q_{1}^{B}}} .
$$

Solving, we learn that $\frac{Q_{2}^{B}}{Q_{1}^{B}}=4$, indicating that $p_{2}$ is $4 \times$ more abundant that $p_{1}$ in sample $B$. Here, we have 4 unknowns from the 2 proteins, and 3 constraints, one from each of the peptide. By using ratios, $\left(\mathrm{Ex}: R_{j}=Q_{j}^{A} / Q_{j}^{B}\right)$, we reduce the number of unknowns, and can solve to get the extra information. Note that the unit of measurement for $Q_{j}^{A}, Q_{j}^{B}$ is immaterial. For this reason, we always reduce one degree of freedom, typically by adding the constraint $\sum_{j} Q_{j}^{B}=100$, or solving for ratios, as we do here. As long as the number of constraints matches the number of unknowns, we can solve to get the relative abundances of different proteins, possible only with shared peptides.

Consider a second example, a more complex one this time, with 3 proteins $p_{1}-p_{3}$ and 5 peptides $s_{1}-s_{5}$, as shown in Figure 1b. Here, protein $p_{2}$ does not have 


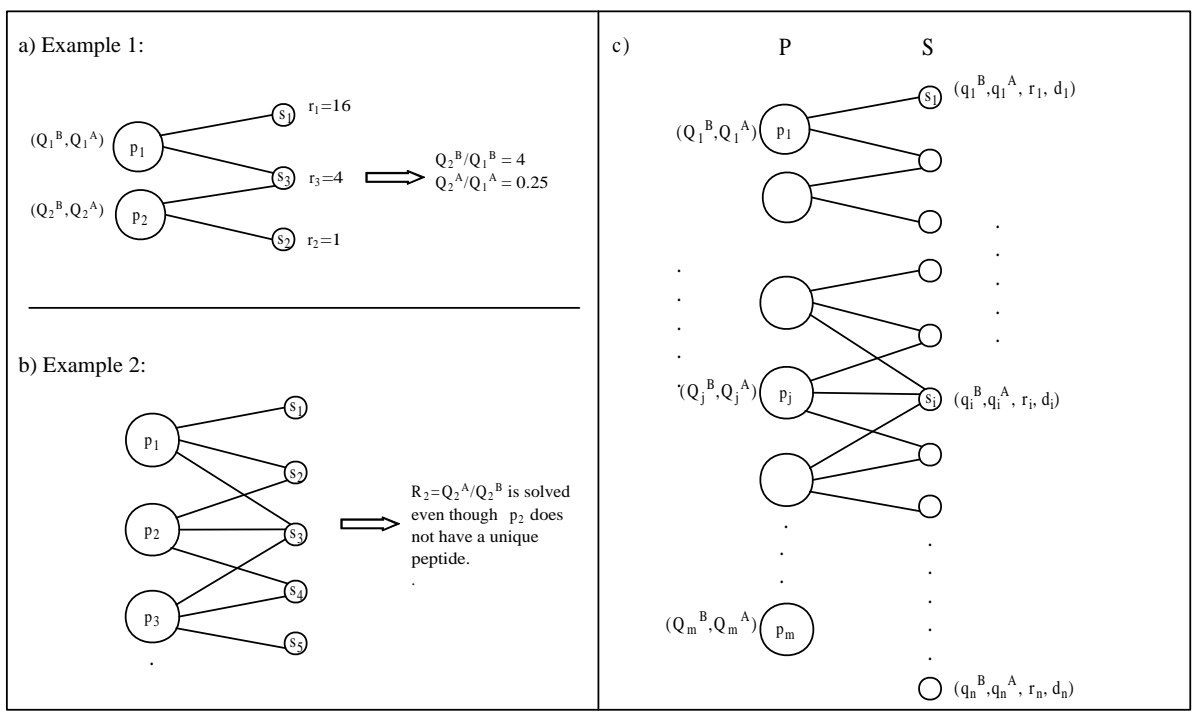

Fig. 1. (a, b) Two examples illustrating our approach for protein quantification via shared peptides. (c) Protein-peptide bi-partite graph $G=(P \cup S, E)$ representing the mapping between $m$ proteins and $n$ peptides.

any unique peptide, and would normally be discarded. However, the system has $5+1$ constraints, and 6 unknowns. Therefore, solving the system gives us $Q_{2}^{A}, Q_{2}^{B}$, and therefore, the relative abundance $\frac{Q_{2}^{A}}{Q_{2}^{B}}$ of $p_{2}$.

To summarize, shared peptides provide extra information in protein quantification. Under certain conditions, they allow us to a) compute relative abundance of a protein even when it does not contain a unique peptide, and b) compute relative abundance values of two different proteins in a sample. To our knowledge, this is the first paper to exploit shared peptides in this manner. However, the simple idea is confounded by the realities of missing data, and error in experiments. Here, we lay out the theoretical foundations and practical considerations in determining when the shared peptide abundances can be used reliably. We show that the solvability must depend upon the topological properties of the peptide-protein relationships as well as numerical properties of the experimentally determined intensity values. It is often the case that interesting cases cannot be resolved because of missing data, or numerical instability.

As an extension to our approach, we also consider some intrinsic properties of peptides. Informally, define the detectability of a peptide as the probability that it will be detected via MS, when the parent protein is expressed. We propose an alternative formulation that estimates the peptide detectabilities in addition to absolute and relative abundances of proteins when appropriate data is available.

Furthermore, we suggest two improvements to increase the number of cases that can be solved. First, we describe a algebraic technique based on singular 
value decomposition to make robust inferences for numerically ill-conditioned systems. Recent results have shown that detectability is indeed an intrinsic characteristic of peptides that can be computed in independent experiments, and maintained for future use [1]. We also point out that incorporating detectabilities as known variables in our formulation, it is possible to solve a much larger number of cases.

In Section 2, we describe the theoretical and empirical considerations for shared peptide analysis. In Section 3.1, we validate our approach with extensive simulations. We apply our methods to data from ITRAQ experiments comparing an Arabidopsis model of root-knot infection versus wild-type in Section 3.2. Our results elucidate the relative abundance among different members of a family in over 55 Arabidopsis protein families.

\section{Protein quantification via shared peptides}

We represent the protein quantification data using a bipartite graph $G=(P \cup$ $S, E)$ where $P$ is the set of proteins and $S$ is the set of peptides. For all $p \in P, s \in$ $S,(p, s) \in E$ if and only if peptide $s$ is a substring of the protein sequence $p$. Note that different connected components of $G$ do not influence each other, and we treat each component independently. W.l.o.g, assume that $G$ is connected, and let $|P|=m$ and $|S|=n$. See Figure 1c. Consider the case where only two samples are involved. In many experiments, the abundances are measured before and after a treatment, so we denote the samples as $B$, and $A$. We associate two variables $\left(Q_{j}^{B}, Q_{j}^{A}\right)$ corresponding to the 'before' and 'after' abundance for each protein $p_{j} \in P$. As mentioned earlier, we also add the constraint $\sum_{j} Q_{j}^{B}=100$.

Analogous to proteins, we associate values $q_{i}^{B}, q_{i}^{A}, r_{i}$ with each peptide $s_{i} \in$ $S, i=1: n$ where $r_{i}=q_{i}^{A} / q_{i}^{B}$ denotes the ratio of the peptide $s_{i}$ abundance between samples. It is possible to generalize the representation for the data with more than two samples. While this abstraction hides many of the complexities of protein quantification via mass spectrometry, it is useful to present our approach which can be applied to many different quantification protocols, including labeled and label-free approaches.

Key to our computation are equations that connect all proteins $p_{j}$ which contain a single peptide $s_{i}$. In the absence of experimental error, the abundance values must satisfy the following $n+1$ constraints over $2 m$ variables.

$$
\begin{aligned}
& \sum_{\left(p_{j}, s_{i}\right) \in E} Q_{j}^{A}-r_{i} \times \sum_{\left(p_{j}, s_{i}\right) \in E} Q_{j}^{B}=0 \text { for all } s_{i} \in S \\
& \sum_{j} Q_{j}^{B}=100
\end{aligned}
$$

With no errors, we can solve this equation uniquely as long as $n+1 \geq 2 m$. To incorporate errors, we consider a linear-programming formulation that minimizes the total error. (See Figure 2, $\mathrm{F}_{1}$ formulation.)

Note that the ratios are not symmetric about 1 , so we always choose a constraint where the ratio contribution is greater than 1 . To simplify notation, we 


\begin{tabular}{|c|c|c|c|}
\hline & Input & Output & Formulation \\
\hline $\mathrm{F}_{1}$ & $r_{i}, \forall s_{i} \in S$ & $\begin{array}{l}Q_{j}^{B}, Q_{j}^{A} \\
\forall p_{j} \in P\end{array}$ & $\begin{array}{ll}\min & \sum_{i=1}^{n}\left|\varepsilon_{i}\right| \\
\text { s.t. } & \sum_{p_{j} \in P} Q_{j}^{B}=100 \\
& \varepsilon_{i}=\sum_{\left(p_{j}, s_{i}\right) \in E} Q_{j}^{A}-r_{i} \times \sum_{\left(p_{j}, s_{i}\right) \in E} Q_{j}^{B} \forall s_{i} \in S, r_{i} \geq 1 \\
& \varepsilon_{i}=r_{i} \times \sum_{\left(p_{j}, s_{i}\right) \in E} Q_{j}^{A}-\sum_{\left(p_{j}, s_{i}\right) \in E} Q_{j}^{B} \forall s_{i} \in S, r_{i} \leq 0 \\
& Q_{j}^{B} \geq 0, Q_{j}^{A} \geq 0 \\
\forall p_{j} \in P .\end{array}$ \\
\hline $\mathrm{F}_{2}$ & $\begin{array}{l}q_{i}^{B}, q_{i}^{A}, r_{i} \\
\forall s_{i} \in S\end{array}$ & $\begin{array}{l}Q_{j}^{B}, Q_{j}^{A}, \\
\forall p_{j} \in P \\
\\
d_{i}, \forall s_{i} \in S\end{array}$ & $\begin{aligned} \min & \sum_{i=1}^{n}\left|\varepsilon_{i}^{B}\right|+\left|\varepsilon_{i}^{A}\right| \\
\text { s.t. } & \sum_{p_{j} \in P} Q_{j}^{B}=100 \\
& \varepsilon_{i}^{B}=\sum_{\left(p_{j}, s_{i}\right) \in E} Q_{j}^{B}-q_{i}^{B} f_{i}, \forall s_{i} \in S \\
& \varepsilon_{i}^{A}=\sum_{\left(p_{j}, s_{i}\right) \in E} Q_{j}^{A}-q_{i}^{A} f_{i}, \forall s_{i} \in S \\
& Q_{j}^{B} \geq 0, Q_{j}^{A} \geq 0, \quad \forall p_{j} \in P .\end{aligned}$ \\
\hline
\end{tabular}

Fig. 2. Input, output, and computation summary of two LP formulations for protein quantification via shared peptides. a) $\mathrm{F}_{1}$ : A formulation that does not include peptide detectability, and; b) $\mathrm{F}_{2}$ : using peptide detectabilities. We use $f_{i}=1 / d_{i}$ as the reciprocal of detectability to maintain linear constraints.

will also represent the LP formulation in a matrix form as

$$
\min \sum_{i}\left|\varepsilon_{i}\right| \text { where } \varepsilon=\mathcal{A} x-b, x \geq 0
$$

where $x$ is vector of dimension $2 m$, given by $x=\left[Q_{1}^{B}, \ldots, Q_{m}^{B}, Q_{1}^{A}, \ldots, Q_{m}^{A}\right]^{T}$, $b$ is a $(n+1)$-dimensional vector described by $b=[100,0, \ldots, 0]^{T}$, and $\mathcal{A}$ is a $(n+1) \times 2 m$ matrix. While this LP is not in standard form, it can easily be transformed into one.

The formulation of the linear program is natural in that the LP seeks for protein abundances that optimally fit the observed peptide ratios. Nevertheless, it raises questions about our confidence in the estimates of $Q_{j}$. Note first that a low value for the objective does not necessarily result in robust estimates of $Q_{j}$. Consider an under-determined system, where $n+1<2 m$. By setting an arbitrary subset of $2 m-(n+1)$ variables to 0 , and solving for the remaining, we obtain multiple solutions, each with 0 error. A simple illustration of this is found in the notion of symmetric proteins. Define proteins $p_{1} \in P$ and $p_{2} \in P$ 
as symmetric if and only if the set of incident peptides $S_{1}=\left\{s \mid\left(p_{1}, s\right) \in E\right\}$ and $S_{2}=\left\{s \mid\left(p_{2}, s\right) \in E\right\}$ are identical. Two symmetric proteins imply two identical columns in $\mathcal{A}$, which means that any linear combination of abundances for these 2 proteins will lead to an identical solution. Certainly, we can solve this problem as a special case: simply merge the two identical columns (proteins) into one, effectively reducing $m$. However, more complex dependencies might arise which are harder to detect.

Generalizing, when $\operatorname{rank}(\mathcal{A})<2 m$, we get multiple solutions with zero-error. If however, $\mathcal{A}$ has full column rank, then by parsimony arguments, the LP solution is likely to provide accurate estimates of protein abundance values. Even if the system is full-rank, it might be ill-conditioned, resulting in poor estimates. We define a rank-threshold function to characterize the solvability, a quantity that is closely related to the condition number of the matrix. Start with the singular-value decomposition of $\mathcal{A}$. Using standard approaches, compute matrices $U, V, \Sigma$ such that

$$
\mathcal{A}=V \Sigma U^{T}
$$

$\Sigma$ is a $(n+1) \times 2 m$ diagonal matrix with nonnegative real numbers $\sigma_{1}, \ldots, \sigma_{p}$ on the diagonal. These $p$ diagonal entries describe the singular values of $\mathcal{A}$ in decreasing order of magnitude, where $p \leq \min \{n+1,2 m\}$. $U$ is orthonormal with dimensionality $2 m \times 2 m$, and $V$ is orthonormal with dimensionality $n+1 \times n+1$, respectively. The rank of $\mathcal{A}$ is given by the number of non-zero singular values. We define a related concept, rank-threshold of $\mathcal{A}$ as

$$
\mathcal{R}(\mathcal{A})=\min \left\{t \mid \sigma_{j}>10^{-t} \forall j\right\}
$$

$\mathcal{R}(\mathcal{A})=t$ being low implies that all its singular values are large $\left(\geq 10^{-t}\right)$, implying that estimates of protein abundance values should be robust. In our experiments, we will show that the rank-threshold is a good way to characterize the reliability of the final solution.

Robust estimates for ill-conditioned systems: This formalism allows us to distinguish high rank systems $\mathcal{A}$ for which we can estimate protein abundance reliably, but it also provides a handle into under-determined systems. Using our notation, we can describe an under-determined system as one in which $\mathcal{R}(\mathcal{A})$ is high. Specifically, $\mathcal{R}(\mathcal{A})=\infty$ implies the case when some of the singular values are 0 . For a rank threshold $t$, define the $\operatorname{rank}_{t}$ of $\mathcal{A}$ as

$$
\operatorname{rank}_{t}(\mathcal{A})=\max \left\{j \mid \sigma_{j}>10^{-t}\right\}
$$

This 'thresholded' rank allows us to get the true dimensionality of a system for which we could get robust results. For all $j$, let $U_{j}$ denote the $2 m \times j$ matrix formed by taking the first $j$ columns of $U$ (corresponding to the dominant singular values). Likewise, let $V_{j}$ denote the matrix formed by the first $j$ columns of $V$, and $\Sigma_{j}=\operatorname{diag}\left[\sigma, \ldots, \sigma_{j}\right]$. This implies that if $\operatorname{rank}_{t}(\mathcal{A})=k$, then

$$
\mathcal{R}\left(\mathcal{A} U_{k}\right)=\mathcal{R}\left(V_{k} \Sigma_{k}\right)=t
$$


We choose $B=\mathcal{A} U_{k}$, and solve the linear program for the $k$ dimensional vector $y$

$$
\min \sum_{i}\left|\varepsilon_{i}\right| \text { where } \varepsilon=\mathcal{B} y-b, U_{k} y \geq 0
$$

Note that $\mathcal{R}(B)=t$ implying that the estimates of $y$ are robust. The reason to keep $y$ unconstrained, but impose $U_{k} y \geq 0$ is the following: The values $y$ cannot be interpreted directly, but can be used to retrieve protein abundance values $x$ by solving

$$
x=U_{k} y
$$

Our constraints ensure that the protein abundance values are non-negative.

\subsection{Incorporating peptide detectability:}

Here we consider an alternative formulation that builds on different assumptions to improve robustness to measurement errors and potentially greatly increase the numbers of components that can be solved. Assuming one is able to estimate the absolute peptide abundances $q_{i}^{B}$ and $q_{i}^{A}$ (as previously described [2]), this formulation allows one to relate the absolute peptide abundance with the total abundance of its parent proteins and thus make inferences about peptide detectabilities in addition to relative protein abundances.

We define the detectability of a peptide $s_{i}$ as a quantity $d_{i} \in[0,1]$ that relates peptide abundance to the abundances of its parent proteins. In the absence of experimental error, for each peptide $s_{i} \in S$,

$$
\begin{aligned}
& q_{i}^{B}=d_{i} \times \sum_{\left(p_{j}, s_{i}\right) \in E} Q_{j}^{B} \\
& q_{i}^{A}=d_{i} \times \sum_{\left(p_{j}, s_{i}\right) \in E} Q_{j}^{A} .
\end{aligned}
$$

In dealing with errors, we use a linear programming formulation that is similar to $\mathrm{F}_{1}$, but with $2 n+1$ constraints and $2 m+n$ variables. (See Figure $2, \mathrm{~F}_{2}$ formulation.) We use $f_{i}=\frac{1}{d_{i}}$ as the reciprocal of detectability to maintain linearity of equations. The previous discussion regarding reliable estimates of abundance values is unchanged from the previous section.

The ITRAQ data does not provide peptide abundance values that can be used directly for $\mathrm{F}_{2}$. However, recent developments indicate that the absolute peptide abundances can be experimentally estimated [2]. Also, recent results have shown that peptide detectabilities can be reliably estimated with very little variability across mass spectrometry runs $[1, ?]$. This observation is especially important in $\mathrm{F}_{2}$. The knowledge of peptide detectabilities implies $2 m$ variables instead of $2 m+n$ and greatly increases the number of cases that can be solved.

\section{Results}

Data-set: We choose an Arabidopsis model of root-knot nematode infection. The root-knot nematodes are worm-like, microscopic plant-parasites that infect 
a multitude of plants, including all major crops, turf, and many ornamental plants. The diversity and extent of infection makes it economically significant to explore. The typical mode of infection is via the root. The female nematode lays its eggs at the root tip. The juveniles infect via the root tip, and move up. Inside, they manipulate the cellular machinery to create specialized feeding cells, which grow and multi-nucleate, but do not divide, eventually forming giant cells that provide nutrients to the parasite $[3,10]$. As the nematodes exploit the Arabidopsis cellular machinery to create the giant cell phenotype, an analysis of proteins that are differentially expressed in infected versus non-infected host cells can help elucidate the underlying mechanism [4]. As the Arabidopsis genome is sequenced, with extensive annotation on the known genes and pathways, it is an appropriate model for the host.

An ITRAQ method was used to collect protein abundance information. A brief overview of the method is given here (See [11] for details). The samples are enzymatically digested into short peptides. Peptides from different samples are $N$-terminally covalently labeled with tags of different mass, but then pooled and analyzed together using tandem mass spectrometry. Each spectrum contains both the fragment masses used to identify the peptide, and the intensities of the differential tags for abundance computation. In our terminology, for every peptide $s_{i}$, we read the intensities of the two tags as $q_{i}^{A} q_{i}^{B}$, and compute the ratio $r_{i}=q_{i}^{A} / q_{i}^{B}$, which approximates the ratio of the peptide abundance values in the two samples.

Our data-set is a collection of 118,426 spectra, encoding 27,728 peptides mapping onto 8,584 protein sequences. Each protein is mapped to at least one peptide and vice versa. The number of peptides mapping to a protein sequence varies considerably, ranging from from 1 to 59. The distribution of the number of peptides per protein, is shown in Figure 3. Close to half of the proteins $(4,145$

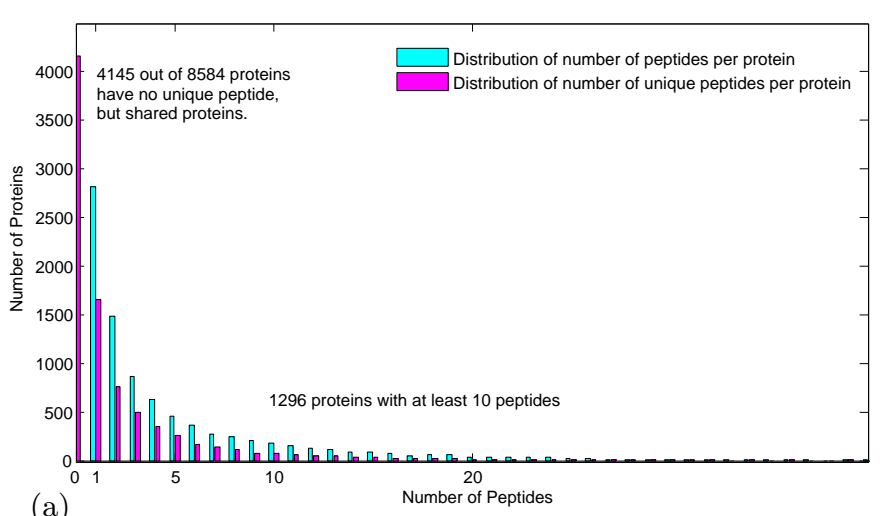

(a)

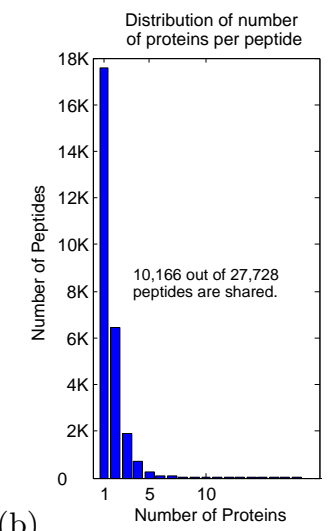

Fig. 3. Mapping of peptides to proteins in Arabidopsis root-knot nematode infection ITRAQ data. (a) Distribution of the number of peptides and unique peptides per protein. (b)Distribution of the number of proteins per peptide. 
out of 8,584 ) do not have a unique peptide. The number of unique peptides per protein range from 0 to 51 . The distribution of the number of unique peptides per protein is shown in Figure 3. Likewise, there is tremendous spectral redundancy among peptides, with the number of spectra encoding a peptide ranging from 1 to 975 . Close to half of the peptides $(10,166)$ are shared by multiple protein sequences. We reduce the data by merging symmetric peptides, or peptides that belonged to an identical subset of proteins. The redundancy helps understand the measurement error, and the merging removes artificial dimensionality, giving a better measure of rank, and rank-threshold. Likewise, we also merge the symmetric proteins for reasons mentioned earlier.

After merging, we obtain a protein-peptide bi-partite graph $G=(P \cup S, E)$, where $|P|=6,998,|S|=8,069,|E|=13,055$. $G$ has 4119 connected components projecting onto 257 non-isomorphic topologies with size ranging from 2 to 127 . In this study, we consider only the 1190 non-trivial components, with at least 2 proteins.

In addition to testing on this data, we also perform a series of controlled experiments by simulating data-sets based on the topologies of the Arabidopsis data-set.

Generation of simulation data: We start with 257 topologically distinct (nonisomorphic) components of the Arabidopsis data, and generated 100 data-sets from each topology with different values ${ }^{3}$. For each component, we do the following:

1. Sample protein amounts $\boldsymbol{Q}^{B}=\left[Q_{1}^{B}, \ldots, Q_{m}^{B}\right]$ at random from the collection of ITRAQ tag intensities.

2. Generate ratio $R_{j}$ by sampling from a $\log$-normal $\mathrm{N}\left(0, \sigma_{R}\right)$ distribution. $\sigma_{R}$ is set to 0.7 which is the estimated standard deviation of the log peptide ratios in the Arabidopsis data. Compute $Q_{j}^{A}=R_{j} Q_{j}^{B}$.

3. For each peptide $s_{i}$, generate $d_{i}$ uniformly from $(0,1]$, and compute $q_{i}^{A}=$ $d_{i} \sum_{\left(p_{j}, s_{i}\right) \in E} Q_{j}^{A}, q_{i}^{B}=d_{i} \sum_{\left(p_{j}, s_{i}\right) \in E} Q_{j}^{B}$. When detectabilities are not incorporated, choose $d_{i}=1$.

4. Compute peptide ratios $r_{i}$, and perturb according to a log-normal $N(0, \sigma)$, over a range of values $\sigma$. Denote $\sigma$ as perturbation level.

We consider the system of constraints for each data-set.

Once the data is generated, only the peptide ratios $r_{i}$ are used as inputs. The linear programs are solved for $\boldsymbol{Q}^{B^{\prime}}=\left[Q_{1}^{B \prime}, \ldots, Q_{m}^{B \prime}\right]$, and $\boldsymbol{Q}^{A \prime}=\left[Q_{1}^{A \prime}, \ldots, Q_{m}^{A \prime}\right]$ using ILOG OPL Development Studio 6.14 . The reliability of the estimates is tested using three measures.

Validation statistics: Recall that the value of the optimized objective is a weak indicator of the quality of results. For the simulations, as the protein abundances

\footnotetext{
${ }^{3}$ Simulation data - http://www.cse.ucsd.edu/ bdost/downloads/SimData.zip

${ }^{4}$ Source code (C\#) - http://www.cse.ucsd.edu/ bdost/downloads/PQPLinearProg.zip
} 
are known, we can compute the error in the estimate as the protein-abundancedistance, PAD:

$$
\operatorname{PAD}\left(\boldsymbol{Q}^{B \prime}, \boldsymbol{Q}^{B}\right)=\frac{\left\|\boldsymbol{R}^{B}\right\|_{1}}{m}
$$

where $\boldsymbol{R}^{\boldsymbol{B}}=\left[\ln \frac{Q_{i}^{B \prime}}{Q_{i}^{B}}\right]$. While any norm can be used as a valid measure of distance, the choice of the 1-norm, averaged over the dimensions can be loosely interpreted as average fold difference between actual and estimated protein abundances. The true protein abundances are not available for the Arabidopsis ITRAQ data, so we compute an indirect measure LRD, defined as

$$
\operatorname{LRD}(\boldsymbol{r}, \boldsymbol{r} \mathbf{\prime})=\frac{\left\|\boldsymbol{r}-\boldsymbol{r}^{\prime}\right\|_{1}}{n}
$$

where $\boldsymbol{r}=\left[\ln \left(r_{1}\right), \ldots, \ln \left(r_{n}\right)\right]$ is the vector of experimental peptide log-ratios for the $n$ peptides, and $\boldsymbol{r}^{\prime}$ describe the peptide log ratios computed by using the estimated protein abundances. Intuitively, if the protein abundance estimates are accurate, the computed peptide log-ratios should match the experimental log-ratios. In a similar way, we compute the peptide log detectability distance LDD as the average 1-norm of the logs of detectabilities. We use PAD, LRD, and LDD to test performance on simulations.

\subsection{Results of Simulation}

As the reliability of the estimates depend upon rank of $\mathcal{A}$, we loosely group each of $100 \times 257$ simulated systems into three categories according to $\operatorname{rank}(\mathcal{A})$ for a fixed rank-threshold $t$, as follows:

Category I : $\operatorname{rank}_{t}(\mathcal{A})=2 m \leq n+1$ (Over-determined, full-rank systems). Category II: $\operatorname{rank}_{t}(\mathcal{A})<2 m \leq n+1$ (Ill-conditioned systems).

Category III : $\operatorname{rank}_{t}(\mathcal{A}) \leq n+1<2 m$ (Under-determined systems).

At rank-threshold 1, we obtain 1074, 3926, and 20700 systems in Categories I, II, and III for the $F_{1}$ simulation, and similar distributions for $F_{2}$. As the rankthreshold is increased, some of the Category II systems move into Category I (Table 1). Additionally, the performance of under-determined systems is uniformly worse than the other two (data not shown). Therefore, we will focus on Category I evaluation using different rank-thresholds. For each category, and each validation statistic, we compute cumulative-probability as the fraction of systems in that category that achieve a certain distance or lower. The ideal case is when the cumulative probability is 1 at distance 0 .

In the absence of noise, we achieve the ideal case, zero PAD and LRD, for all Category I systems at rank-threshold 4. As noise is introduced to data and less stringent rank-thresholds is used, we deviate from the ideal case. Figure 4a shows the cumulative probability distribution against PAD, and LRD at perturbation level 0.01. Out of 1074 Category I systems at rank-threshold 1, 75\% have PAD 
Table 1. Simulation Category I systems grouped according to their rank-thresholds.

\begin{tabular}{|c|c|c|c|c|c|}
\cline { 2 - 6 } \multicolumn{1}{c|}{} & \multicolumn{4}{c}{$\mathcal{R}(\mathcal{A})$} \\
\multicolumn{1}{c|}{} & 1 & 2 & 4 & 8 & 16 \\
\hline$F_{1}$ - Category I & $1074(4.2 \%)$ & $2514(9.8 \%)$ & $3044(11.8 \%)$ & $3980(15.5 \%)$ & $4388(17.1 \%)$ \\
\hline$F_{2}$ - Category I & $663(2.6 \%)$ & $2251(8.8 \%)$ & $2955(11.5 \%)$ & $3104(12.1 \%)$ & $4989(19.4 \%)$ \\
\hline
\end{tabular}

error of less than 0.16 , and an LRD error of less than 0.01 . The performance degrades for higher rank-thresholds. Note that in all cases, the optimized objective is very close to $0\left(\sim 10^{-4}\right)$. However, in the ill-conditioned and under-determined systems, multiple solutions will lead to a low-error solution, and an arbitrarily picked solution will have high PAD and LRD error. The performance also degrades with an increase in perturbation error. Figure $4 \mathrm{~b}$ plots the cumulative ratio for Category I systems at rank-threshold 1 under increasing perturbation levels. Thus while $93 \%$ of systems show LRD of at most 0.1 at perturbation 0.01 , the number falls to $55 \%$ at perturbation 0.15 .

(a)
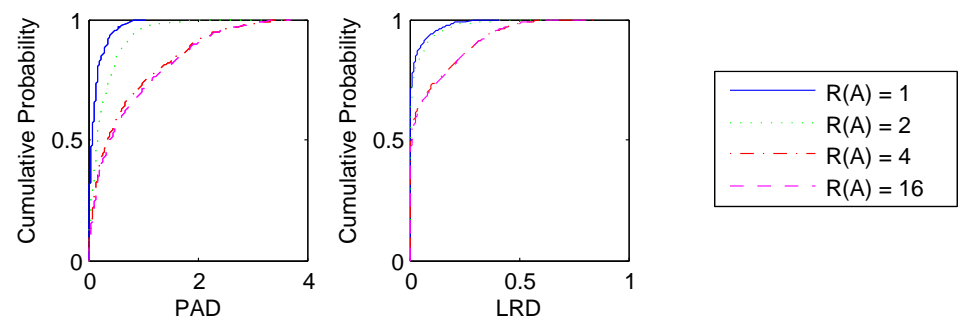

(b)
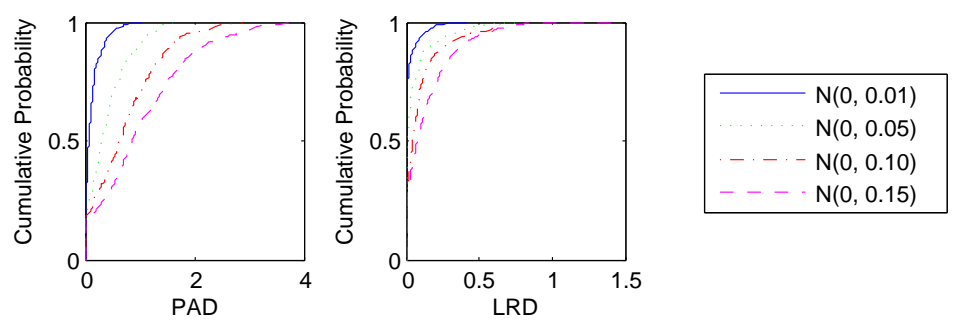

Fig. 4. Simulation results using $F_{1}$ formulation. Cumulative probability of PAD and LRD for Category I systems (a) perturbation level 0.01, but different rank-thresholds (b) at rank-threshold 1, but different perturbation levels. In all cases, we measure the fraction of systems that were estimated within a certain distance.

Ill-conditioned systems We identified a number of Category II systems where the rank-threshold was poor, but only because a small number of singular values were close to 0 . For example, in a simulation with perturbation level 0.05 , we observed 339 systems for which fewer than 3 singular values were at most $10^{-16}$, and $\operatorname{rank}_{1}(\mathcal{A}) \geq 2 m-3$ (remaining s.v. $\geq 10^{-1}$ ). Our results show that the 
revised LP, suggested for ill-conditioned systems in Section 2, indeed provides better estimates of protein abundance values of these systems. (See Figure 5.) For example, over $88 \%$ of the systems from the revised LP achieve an LRD of 0.25 or better, compared to $65 \%$ from the original formulation.
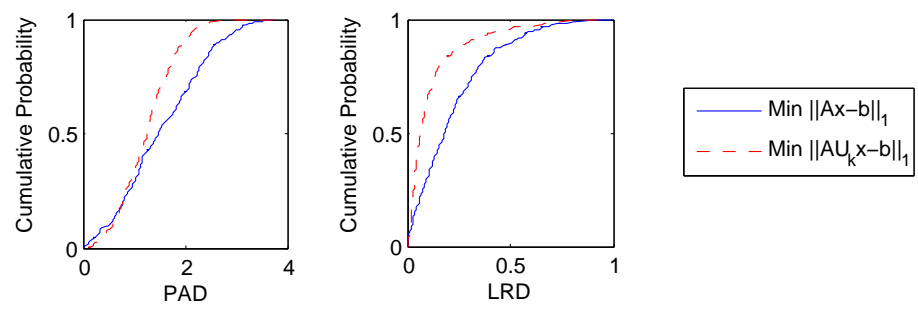

Fig. 5. Improved estimates of protein abundance values using SVD based projection on Category II (over-determined but ill-conditioned systems).

Peptide detectabilities A similar behavior is observed during estimation of peptide detectabilities (Figure 6a,b). The performance of PAD, and LRD surprisingly does not change with the addition of an extra $n$ unknowns (also, $n$ new constraints get added). We also plot the performance of detectability estimates. As expected, the performance is acceptable for low rank-threshold systems and low perturbations, but degrades for higher rank-thresholds, and higher perturbation levels. The detectability estimates are robust as well, and degrade in a similar manner.

\subsection{Arabidopsis ITRAQ data}

We focus on the 1190 non-trivial systems from the ITRAQ data comparing infected samples to non-infected ones. ITRAQ data is not appropriate to get peptide abundance values reliably, so we only use the $F_{1}$ formulation on this data-set. The distribution of systems in different rank categories is described in Figure 7a. A total of 99 systems fall into Category I with the most stringent rank-threshold 1, covering 219 proteins and 357 peptides. In addition to relative protein abundances, we estimate abundance ratios across samples for 4 proteins which do not have a unique peptide.

As actual protein abundances are not known, we use LRD to evaluate the estimates. The LRD statistic of different categories is as expected with this group performing better than the other groups. Within the 99 systems, 79 have LRD smaller than $10^{-1}$, and 55 have LRD smaller than $10^{-4}$. The list of systems, constituent peptides, and protein abundance values are shown in online supple- 

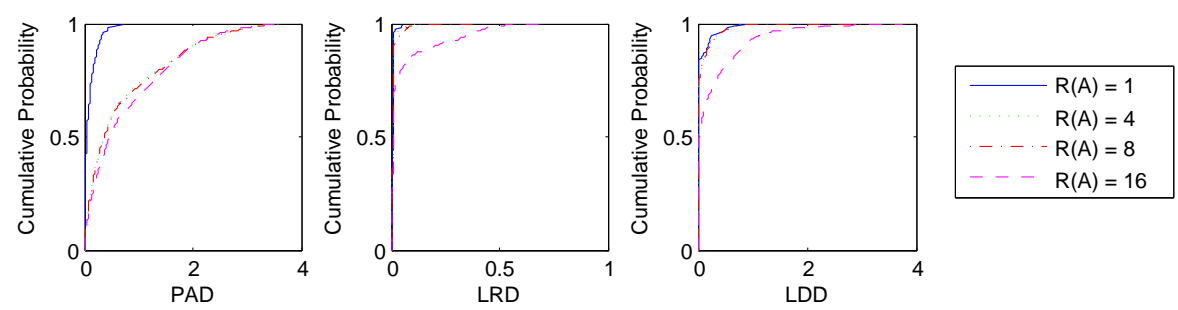

(a)
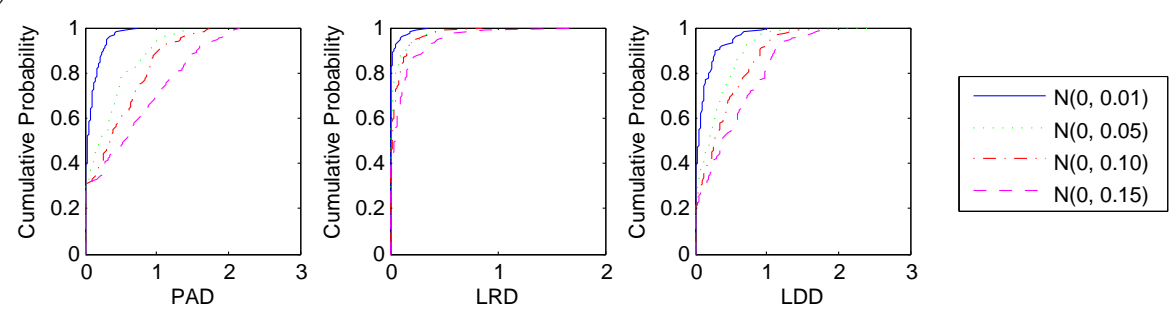

(b)

Fig. 6. Simulation results using $\mathrm{F}_{2}$ formulation. Cumulative probability of PAD, LRD and LDD for Category I systems (a) at different rank-thresholds (b) at rank-threshold 1 , but different perturbation levels. In all cases, we measure the fraction of systems that were estimated within a certain distance.

mental data ${ }^{5}$. Here, we cherry-pick a few representative examples that point to the differential expression of individual sequences in response to the infection.

(a)

\begin{tabular}{|c|c|c|}
\hline $\mathcal{R}(\mathcal{A})$ & Cat. I & Cat. II \\
\hline 1 & $99(8.3 \%)$ & $191(16 \%)$ \\
2 & $249(20.9 \%)$ & $41(3.4 \%)$ \\
4 & $276(23.2 \%)$ & $14(1.2 \%)$ \\
8 & $277(23.3 \%)$ & $13(1.1 \%)$ \\
16 & $282(23.7 \%)$ & $8(0.7 \%)$ \\
\hline
\end{tabular}

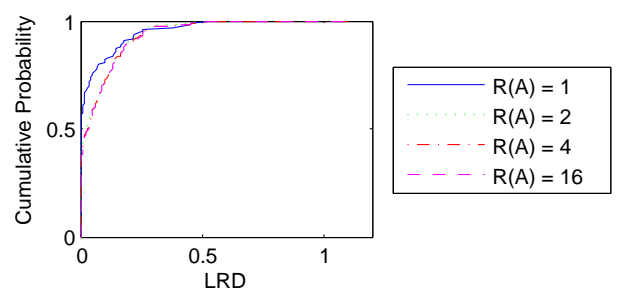

Fig. 7. Arabidopsis root-knot nematode infection ITRAQ data. (a) Number of systems in Category I and II at different rank-thresholds. (b) Empirical cumulative probability distribution of LRD for Category I systems at different rank-thresholds.

$\mathrm{Ca}^{2+}$ ATPases: One of the systems is encoded by 3 proteins from the P-type $\mathrm{Ca}^{2+}$ ATPase super-family involved in $\mathrm{Ca}^{2+}$ transport. The three members are the plasma-membrane bound AT5G57110 (ATPase 8), AT4G2990 (ATPase 10),

\footnotetext{
${ }^{5}$ Supplemental data -

http://www.cse.ucsd.edu/ bdost/downloads/Recomb09SuppData.pdf
} 


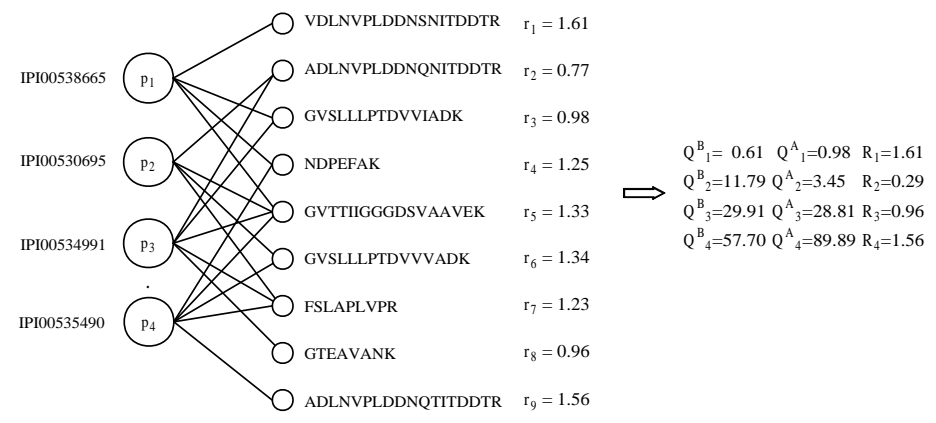

Fig. 8. PhosphoGlycerate kinase family members sharing peptides.

and AT3G21189(ATPase 9). Earlier reports have suggested that ATPase8,10 are co-expressed evenly over all vegetative tissues, while ATPase 9 is expressed almost exclusively in pollen [9]. In our data, the 3 form a confected component with 6 peptides, and our analysis showed the relative wild-type expression of the 3 to be $34.3 \%, 57.5 \%, 8.22 \%$ respectively, confirming this observation. Further, we find that ATPase 8 is $3 \times$ over-expressed in the infected state.

Profilins: The Profilin family encodes proteins that regulate actin cytoskeleton formation. Five profilins are known. The two that are identified in our data (PRF-1,2) are constitutively expressed in all vegetative organs, and a regulatory element in their first intron is suspected to mediate this expression, differentiating them from the other Profilins [5]. In our data-set, the two are in a component with 3 peptides, one shared. Our analysis shows that Profilin-2 has only (13\%) of the total abundance, and is further reduced two-fold upon infection.

Cinnamyl-alcohol dehydrogeneases: A number of genes in Arabidopsis have been annotated as part of the CAD family, an assertion which has subsequently been challenged, pointing instead to the central role of two members (AtCAD4, and AtCAD5) in the CAD metabolic network. These two molecules have expression patterns consistent with lignification at stem tissues. Interestingly, expression was also observed in various non-lignifying zones (e.g. root caps) indicative of a possible role in plant defense [7]. Our results have a single connected component with AtCAD4,5 and 3 peptides. The analysis shows that both proteins are equally abundant, with AtCAD5 (AT4G34230) at 56\% in non-infected cells. However, AtCAD5 is significantly $(1.5 \times)$ over-expressed, while AtCAD4 (AT3G19450) is $2 \times$ under-expressed.

Phosphoglycerate kinases: Phosphoglycerate kinases have been previously shown to be differentially expressed during defense response of Arabidopsis [6]. In our data, four proteins from this family are in a component with nine peptides as shown in Figure 8. In this example, along with the relative protein abundances, we also compute the abundance ratio across-sample for IPI00530695 even though it does not have a unique peptide. Our analysis suggests that both IPI00538665 and IPI00535490 are $1.5 \times$ over-expressed, but IPI00535490 is much more abun- 
dant in both samples. IPI00530695 is $3 \times$ under-expressed while the abundance of IPI00534991 does not change.

\section{Discussion}

The extent of peptide sharing in proteomics is under-estimated; consequently, shared peptides, and proteins with non-unique peptides are typically discarded, corresponding to as much as $50 \%$ of the data in our experience. As mass spectrometry based protein quantification becomes routine, shared peptide analysis will be increasingly important. Our results are the first to show that a careful analysis not only helps in recovering abundance values of some of these proteins, but also helps quantify the relative levels of different proteins. These acrossprotein relative abundance computations can help elucidate these differential regulation of the proteins from a family. We investigate topological and numerical considerations in estimating reliability of our computations. Nevertheless, the final quality of the results does depend upon the accuracy of the experimental abundance computations $[2,8]$. As the mass spectrometers become more accurate, experimental variation in relative abundance computations will decrease, increasing the power of our methods. In a similar fashion, the estimation of peptide detectabilities is in an early stage of development. Our results attest to the viability of using shared peptides for detectability computation, but also point to the importance of detectability values in extending the scope of shared peptide analysis. The model's ability to automatically estimate peptide detectabilities may result in an ongoing cycle of self-refinement where different systems resulting from different experimental conditions may allow one to continuously expand the set of known detectabilities, which in turn would allow for the resolution of more complicated systems. In fact, we note that this progressive convergence towards an extensive database of peptide detectabilities may even allow one to learn more about systems that were previously not solvable in a given experiment by adding information from different or even additional targeted experiments aimed at estimating the necessary detectabilities.

A final contribution of this paper is the use of novel evaluation methods for shared peptide computations. Clearly, different algorithms can be used to optimize the error in estimation, including non-linear optimization and other machine learning approaches. We have experimented using simulated annealing approach with a non-linear cost function that minimizes the absolute sum of differences between observed and expected peptide ratios. While such approach is more time consuming, it provides better estimates for systems with unbalanced protein abundances as linear programming formulation is biased towards the error terms associated with the more abundant proteins. Details of that study will be discussed somewhere else. This paper describes a systematic simulation based framework to compare, and develop improved methods for shared peptide analysis. 


\section{Acknowledgments}

The research was supported by the National Center for Research Resources of NIH via grant P-41-RR24851.

\section{References}

1. P. Alves, R.J. Arnold, M.V. Novotny, P. Radivojac, J.P. Reilly, and H. Tang. Advancement in protein inference from shotgun proteomics using peptide detectability. Pac Symp Biocomput, pages 409-420, 2007.

2. M Bantscheff, M Schirle, G Sweetman, J Rick, and B Kuster. Quantitative mass spectrometry in proteomics: a critical review. Anal Bioanal Chem, 389(4):10171031, Oct 2007.

3. An Advanced Treatise on Meloidogyne: Volume I. North Carolina State University Graphics, 1985

4. F. Jammes, P. Lecomte, J. de Almeida-Engler, F. Bitton, ML Martin-Magniette, JP. Renou, P. Abad, and B. Favery. Genome-wide expression profiling of the host response to root-knot nematode infection in Arabidopsis. The Plant Journal, 44:447458, August 2005.

5. Y.M. Jeong, J.H. Mun, I. Lee, J.C. Woo, C.B. Hong, and S.G. Kim. Distinct roles of the first introns on the expression of Arabidopsis profilin gene family members. Plant Physiol., 140:196-209, Jan 2006.

6. A M Jones, M H Bennett, J W Mansfield, and M Grant. Analysis of the defence phosphoproteome of arabidopsis thaliana using differential mass tagging. Proteomics, 6(14):4155-4165, Jul 2006.

7. S.J. Kim, K.W. Kim, M.H. Cho, V.R. Franceschi, L.B. Davin, and N.G. Lewis. Expression of cinnamyl alcohol dehydrogenases and their putative homologues during Arabidopsis thaliana growth and development: lessons for database annotations? Phytochemistry, 68:1957-1974, Jul 2007.

8. P Mallick, M Schirle, S S Chen, M R Flory, H Lee, D Martin, J Ranish, B Raught, R Schmitt, T Werner, B Kuster, and R Aebersold. Computational prediction of proteotypic peptides for quantitative proteomics. Nat Biotechnol, 25(1):125-131, Jan 2007.

9. A. Marmagne, M.A. Rouet, M. Ferro, N. Rolland, C. Alcon, J. Joyard, J. Garin, H. Barbier-Brygoo, and G. Ephritikhine. Identification of new intrinsic proteins in Arabidopsis plasma membrane proteome. Mol. Cell Proteomics, 3:675-691, Jul 2004.

10. http://www.nematology.umd.edu/rootknot.html.

11. S. Wiese, K. A. Reidegeld, H. E. Meyer, and B. Warscheid. Protein labeling by itraq: a new tool for quantitative mass spectrometry in proteome research. Proteomics, 7(3):340-350, February 2007. 